\title{
Nanophotonic Neural Probes for in vivo Light Sheet Imaging
}

\author{
Wesley D. Sacher ${ }^{1}$, Xinyu Liu ${ }^{1 *}$, Ilan Felts Almog ${ }^{2 *}$, Anton Fomenko ${ }^{3 *}$, Thomas Lordello ${ }^{2}$, Fu-Der Chen ${ }^{2}$, \\ Homeira Moradi-Chameh ${ }^{3}$, Azadeh Naderian ${ }^{3}$, Michael Chang ${ }^{3}$, Trevor M. Fowler ${ }^{1}$, Taufik A. Valiante ${ }^{3,4,5,2}$, \\ Andres M. Lozano ${ }^{3,4}$, Laurent C. Moreaux ${ }^{1}$, Joyce K. S. Poon ${ }^{2,6}$, Michael L. Roukes ${ }^{1}$ \\ ${ }^{1}$ Division of Physics, Mathematics, and Astronomy, California Institute of Technology, Pasadena, California 91125 , USA \\ ${ }^{2}$ Department of Electrical and Computer Engineering, University of Toronto, 10 King's College Rd., Toronto, Ontario M5S 3G4, Canada \\ ${ }^{3}$ Krembil Research Institute, University Health Network, Toronto, Ontario, Canada \\ ${ }^{4}$ Division of Neurosurgery, Department of Surgery, Toronto Western Hospital, University of Toronto, Toronto, Ontario, Canada \\ ${ }^{5}$ Institute of Biomaterials and Biomedical Engineering, University of Toronto, Toronto, Ontario, Canada \\ ${ }^{6}$ Max Planck Institute for Microstructure Physics, Halle, Germany \\ *Equal Contribution \\ roukes@caltech.edu
}

\begin{abstract}
We present implantable silicon neural probes with nanophotonic waveguide routing networks and grating emitters for light sheet imaging. Fluorescein beam profiles, fluorescent bead imaging, and fluorescence brain imaging in vivo are presented. (c) 2019 The Author(s)

OCIS codes: (170.0170) Medical optics and biotechnology; (130.0130) Integrated optics
\end{abstract}

Light sheet microscopy is a powerful technique for high speed volumetric fluorescence imaging [1,2]. Conventional light sheet microscopes require macroscopic lenses and objectives for excitation and emission, and these must be positioned at $90^{\circ}$ to one another. This has restricted their application to small, quasi-transparent organisms (e.g., larval zebrafish, drosophila embryo, C. elegans) and cleared mammalian brains [2]. Light-sheet microscopy has been extended to applications in vivo in mammalian brains via miniaturized optics to create implantable probes that generate light sheets within tissue. Critical requirements for such an approach are: 1) the light sheet must illuminate tissue parallel to the brain's surface, and 2) the optics must be sufficiently small to permit generation of light within, or in close proximity to, the collection field-of-view. A miniature light-sheet illumination probe employing a fibercoupled GRIN lens and a micro-prism has enabled creation of a light sheet beneath a second GRIN lens used for fluorescence collection, but only generated a single light sheet [3]. Further, the lateral prism dimensions $(\approx 1 \mathrm{~mm})$ required significant tissue removal to permit its insertion. More recently, a chip-scale probe for light-sheet formation had employed a grating coupler (GC) that emits light into a glass spacer block with a metallic slit lens [4]. Here again, only one sheet was generated, and probe thickness $(>100 \mu \mathrm{m})$ was large compared to silicon probes $[5,6]$.

Here, we present implantable, ultra-thin, shank-based, silicon probes employing nanophotonic silicon nitride (SiN) waveguides for light sheet microscopy in vivo. These novel probes are capable of generating five light sheets, and permit fast ( 10ms) switching times between the sheets (limited by external optics). The probe operation has been tested in fluorescein, an agarose block with fluorescent beads, and in vivo in Thy1-GCaMP6s mice.

A schematic of our probes and an illustration of light sheet formation are shown in Figs. 1a-b. Light is coupled onto the chip via fiber-to-chip edge couplers, a SiN waveguide routing network then splits this light into eight waveguides, which route it along the shanks and ultimately deliver it into grating-coupler (GC) emitters (Fig. 1c-d) that emit the light. The GC output divergence along the vertical axis in Fig. 1b is set by the grating strength.
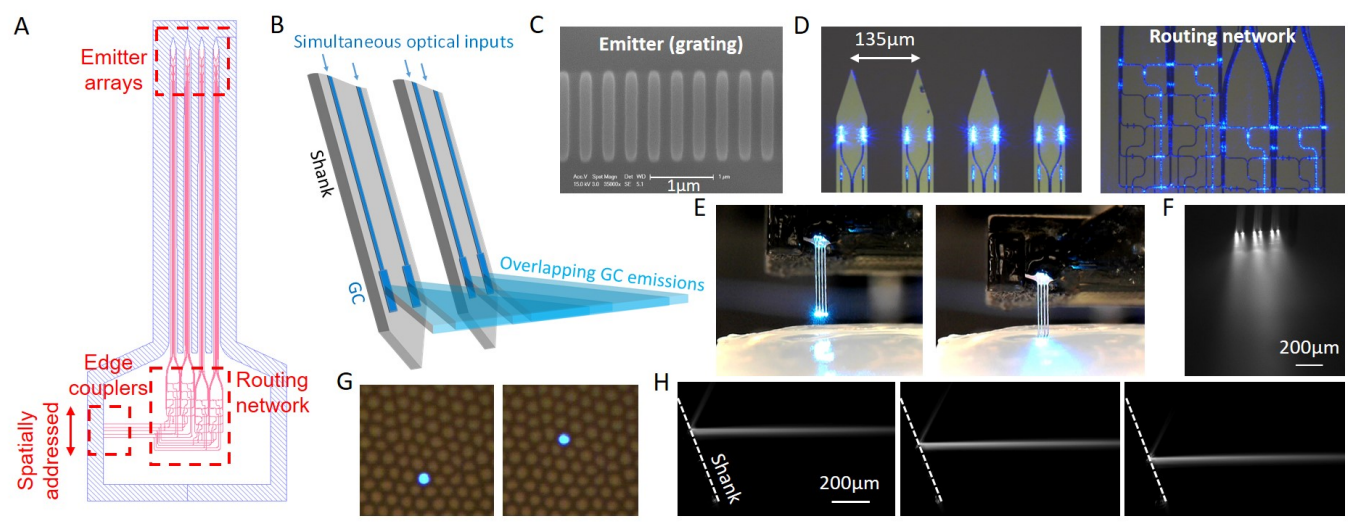

Figure 1. Light sheet neural probes. (a), (b) Illustrations of the probe. Four $3 \mathrm{~mm}$ long shanks on a $135 \mu \mathrm{m}$ pitch have 5 rows of grating couplers (GCs) each capable of emitting a sheet on an $80 \mu \mathrm{m}$ pitch. (c) Scanning electron micrograph (SEM) of a GC. (d) Micrographs of the shanks and routing network with optical input to one of the edge couplers. (e) Photographs of the packaged probe before and after insertion into an agarose block. (f) Top-down micrograph of one of the sheets immersed in fluorescein. (g) Micrographs of the output facet of an image bundle connected to our scanning system showing addressing of different cores. (h) Side micrographs of 3 of the sheets in fluorescein. 

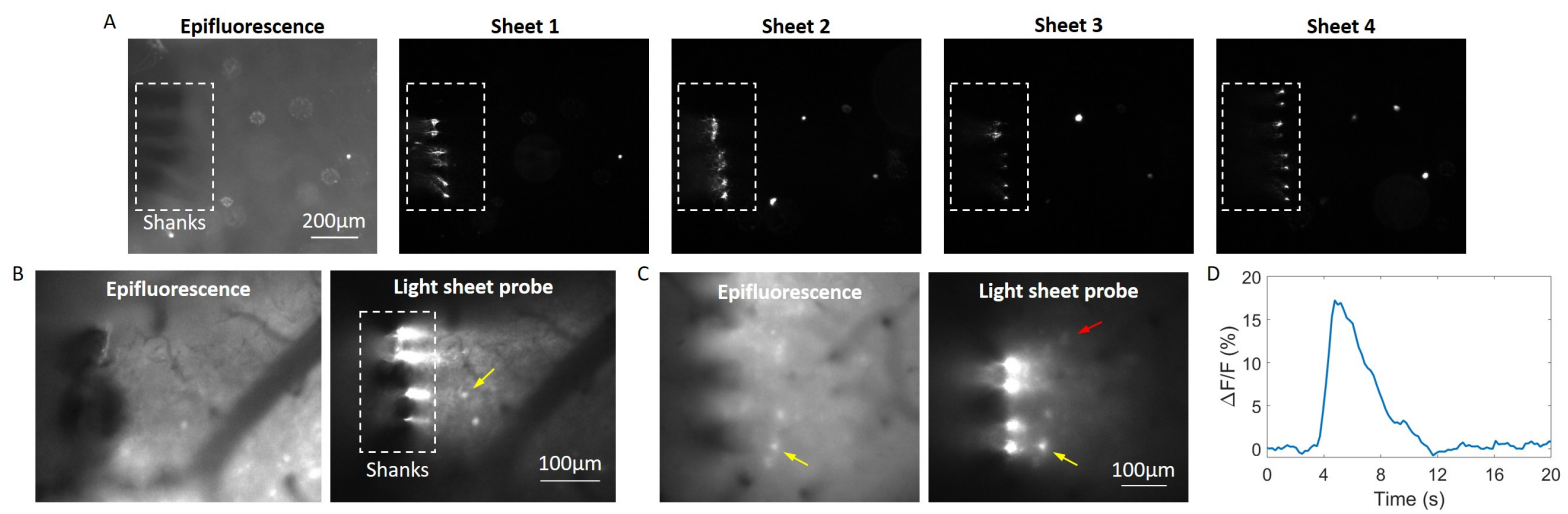

Figure 2. Fluorescent bead and in vivo tests. (a) Imaging of fluorescent beads suspended in an agarose block using epifluorescence microscopy and light sheet illumination from the implanted probe. The epifluorescence image corresponds to the depth plane for sheet 1. (b), (c) in vivo test of the light-sheet probe in the cortex of an anesthetized Thy1-GCaMP6s mouse comparing epifluorescence imaging with probe light-sheet illumination. (d) Time-dependent fluorescence $(\Delta \mathrm{F} / \mathrm{F})$ of the neuron corresponding to the red arrow in (d) (200ms exposure, background fluctuation $\approx 3 \%$ during event). The dashed boxes show the position of the shanks and the point sources within are scattered light from the GCs. The probe variation in (b)-(d) used 4 GCs per sheet. All experimental procedures described in this study were reviewed and approved by the University Health Network Animal Care Committee.

We have previously shown that SiN GCs form narrow beams along this axis, with lateral profiles $\sim 10 \mu \mathrm{m}$ full width at half maximum (FWHM) [5]. Along the horizontal axis of Fig. 1b, the divergence is set by the width of the GC, with narrow (wide) GCs forming high (low) divergence beams. Emission profiles similar to Fig. $1 \mathrm{~b}$ are possible via $\mathrm{GC}$ widths $<1.5 \mu \mathrm{m}$. The overlapping emission of multiple GCs synthesizes a light sheet with uniformity and spatial extent dictated by shank pitch and GC divergence. Similar to the approach in [7], the probe is coupled to a fiber image bundle, and a MEMS mirror-scanning system spatially addresses each on-chip edge coupler via the different cores (Fig. 1g). The probes were fabricated using the methods in [5]; the shanks were $\sim 18 \mu \mathrm{m}$ thick, and the SiN waveguide thickness is $200 \mathrm{~nm}$.

To characterize light sheet uniformity, the probe is immersed in fluorescein (Fig. 1f). In the initial $150 \mu \mathrm{m}$ of propagation, the GC emissions have not yet merged; this results in the observed intensity variations. At propagation distances between $150-350 \mu \mathrm{m}$, intensity variations are $<2.5 \mathrm{x}$ over the $360 \mu \mathrm{m}$ sheet width. In scattering media, the propagation distance at which the GC emissions merge should be closer. The side-micrographs in fluorescein (Fig. 1h) do not accurately represent sheet thickness due to the sheet extending beyond the depth of focus; however, our simulations indicate thicknesses $<10 \mu \mathrm{m}$ FWHM can be achieved. We test fluorescence imaging by inserting the probe into an agarose block containing $10 \mu \mathrm{m}$ diameter fluorescent beads (Fig. 1e) and illuminate it with light sheets oriented parallel to the block's top surface. Induced fluorescence (via 488nm excitation) is collected by an epifluorescence microscope positioned above the probe and agarose block. An electrically-tunable lens attached to the back of the objective provides fast focus adjustment that spans several light-sheet planes. Fig. 2a compares images captured with four light sheets against an epifluorescence image (microscope epi-illumination, with no probe output). Light sheet illumination eliminates out-of-focus beads and demonstrates planar optical sectioning.

Our initial in vivo test of the light-sheet probe is shown in Figs. 2b-c. A probe was inserted into the parietal cortex of an anesthetized Thy1-GCaMP6s mouse [8] at depths $<200 \mu \mathrm{m}$. Fluorescence was collected with an epifluorescence microscope, and a comparison was made between probe-based and epifluorescent illumination. The light-sheet probes enhance the contrast of the neurons; the contrast enhancement is $2-3.5 \mathrm{x}$ for the neurons corresponding to the yellow arrows in Figs. 2b-c. Figure 2d shows the extracted time-dependent fluorescence due to probe illumination from the activity of the neuron marked by the red arrow in Fig. 2(c).

In conclusion, we have developed and validated novel, implantable, nanophotonic light-sheet neural probes that circumvent the incompatibility of conventional light-sheet microscopes with in vivo brain imaging. Combining these probes with GRIN lenses or fiber endoscopes [3,9] for fluorescence collection will enable deep-brain lightsheet imaging. High-speed volumetric functional imaging using multiple sheets is currently being characterized.

\section{Acknowledgments}

We thank Alex Jacob for his advice on GCaMP6 functional imaging.

\section{References}

[1] J. Huisken et al., Science, 305(5686), 1007-1009 (2004).

[2] P. J. Keller and M. B. Ahrens, Neuron, 85(3), $462-483$ (2015).

[3] C. J. Engelbrecht et al., Opt Lett., 35(9), 1413-1415 (2010).

[4] F. Ye et al., in Conference on Lasers and Electro-Optics,

(Optical Society of America, 2016), paper JW2A.142.
[5] E. Segev et al., Neurophotonics 4(1), 011002 (2017).

[6] S. Libbrecht et al., J. Neurophysiol. 120(1), 149-161 (2018).

[7] A. N. Zorzos, et al., Opt. Lett. 37(23), 4841-4843 (2012).

[8] H. Dana et al., PLoS One, 9(9), e108697 (2014).

[9] K. K. Ghosh et al., Nat. Methods 8(10), 871-878 (2011). 